\title{
Erratum
}

Pinki Kumari, R. K. Gupta, Sachin Kumar*, and Maysaa Mohamed Al Qurashi

\section{Erratum to "Conserved vectors with conformable derivative for certain systems of partial differential equations with physical applications"}

https://doi.org/10.1515/phys-2020-0219

received September 27, 2020; accepted November 27, 2020

Abstract: This erratum corrects the typing mistakes of the article "Conserved vectors with conformable derivative for certain systems of partial differential equations with physical applications," published in Open Physics 2020;18(1):164-9, https://doi.org/10.1515/phys-2020-0127.

Keywords: systems of partial differential equations, Lie symmetry, conformable derivative, conservation laws

\section{The dispersive long-wave system}

In ref. [1], the classical dispersive long-wave system is given by

which is used to describe evolution of the horizontal velocity portion of water waves. We present here the correct form of (1) with conformable operator as

$$
\begin{aligned}
& \frac{\partial^{\alpha} u}{\partial t^{\alpha}}=2 u \frac{\partial^{\alpha} u}{\partial x^{\alpha}}-\frac{\partial^{2 \alpha} u}{\partial x^{2 \alpha}}+2 \frac{\partial^{\alpha} v}{\partial x^{\alpha}}, \\
& \frac{\partial^{\alpha} v}{\partial t^{\alpha}}=2 u \frac{\partial^{\alpha} v}{\partial x^{\alpha}}+2 v \frac{\partial^{\alpha} u}{\partial x^{\alpha}}+\frac{\partial^{2 \alpha} v}{\partial x^{2 \alpha}} .
\end{aligned}
$$

* Corresponding author: Sachin Kumar, Department of Mathematics and Statistics, Central University of Punjab, Punjab, India,

Pinki Kumari: Department of Mathematics and Statistics, Central University of Punjab, Punjab, India,

e-mail: yadav.pk1403@gmail.com

R. K. Gupta: Department of Mathematics, Central University of Haryana, Haryana, India, e-mail: rajeshateli@gmail.com
We present the symmetries of the correct version of conformable dispersive long-wave system described in (2) as

$$
\begin{aligned}
& \xi^{1}=\frac{c_{1} t}{\alpha}+c_{2} t^{1-\alpha}, \quad \xi^{2}=\frac{c_{1}}{2 \alpha} x+c_{4} x^{1-\alpha}+\frac{c_{3}}{\alpha} x^{1-\alpha} t^{\alpha}, \\
& \eta_{1}=-\frac{c_{1}}{2} u-\frac{c_{3}}{2}, \quad \eta_{2}=-c_{1} v .
\end{aligned}
$$

Thus, the Lie algebra of (2) is spanned by the following four generators:

$$
\begin{aligned}
& X_{1}=\frac{t}{\alpha} \frac{\partial}{\partial t}+\frac{x}{2 \alpha} \frac{\partial}{\partial x}-\frac{1}{2} u \frac{\partial}{\partial u}-v \frac{\partial}{\partial v}, \quad X_{2}=t^{1-\alpha} \frac{\partial}{\partial t}, \\
& X_{3}=\frac{x^{1-\alpha} t^{\alpha}}{\alpha} \frac{\partial}{\partial x}-\frac{1}{2} \frac{\partial}{\partial u}, \quad X_{4}=x^{1-\alpha} \frac{\partial}{\partial x} .
\end{aligned}
$$

Below, we will present the correct form of conserved 25 vectors.

For the symmetry $X_{1}=\frac{t}{\alpha} \frac{\partial}{\partial t}+\frac{x}{2 \alpha} \frac{\partial}{\partial x}-\frac{1}{2} u \frac{\partial}{\partial u}-v \frac{\partial}{\partial v}$, we obtain

$$
\begin{aligned}
T_{1}^{t}= & c_{1} x^{\alpha-1}\left(-\frac{1}{2} u-\frac{x}{2 \alpha} u_{x}-\frac{t}{\alpha} u_{t}\right) \\
& +c_{2} x^{\alpha-1}\left(-v-\frac{x}{2 \alpha} v_{x}-\frac{t}{\alpha} v_{t}\right), \\
T_{1}^{x}= & 2\left(-\frac{1}{2} u-\frac{x}{2 \alpha} u_{x}-\frac{t}{\alpha} u_{t}\right)\left(c_{1} u-c_{2} v\right) t^{1-\alpha} \\
& -c_{1}\left(\frac{1}{2} u_{x}+\frac{x}{2 \alpha} u_{x x}+\frac{1}{2 \alpha} u_{x}+\frac{1}{\alpha} u_{t x}\right) x^{1-\alpha} t^{\alpha-1} \\
& -\left(-v-\frac{x}{2 \alpha} v_{x}-\frac{t}{\alpha} v_{t}\right)\left(2 c_{1}+2 c_{2} u+c_{2} x^{-\alpha}\right. \\
& \left.+c_{2}(1-\alpha) x^{-\alpha}\right) t^{\alpha-1} \\
& +c_{2}\left(v_{x}+\frac{x}{2 \alpha} v_{x x}+\frac{1}{2 \alpha} v_{x}+\frac{1}{\alpha} v_{t x}\right) x^{1-\alpha} t^{\alpha-1} .
\end{aligned}
$$

For the symmetry $X_{2}=t^{1-\alpha} \frac{\partial}{\partial t}$, we obtain

$$
\begin{aligned}
T_{1}^{t}= & -c_{1} x^{\alpha-1} t^{1-\alpha} u_{t}-c_{2} x^{\alpha-1} t^{1-\alpha} v_{t}, \\
T_{1}^{\chi}= & -t^{1-\alpha} u_{t}\left(c_{1} u-c_{2} v\right) t^{1-\alpha} \\
& -c_{1} t^{1-\alpha} u_{t x} x^{1-\alpha} t^{\alpha-1}+t^{1-\alpha} v_{t}\left(2 c_{1}+2 c_{2} u\right. \\
& \left.+c_{2} x^{-\alpha}+c_{2}(1-\alpha) x^{-\alpha}\right) t^{\alpha-1}+c_{2} v_{t x} x^{1-\alpha} .
\end{aligned}
$$

For the symmetry $X_{3}=\frac{x^{1-\alpha} t^{\alpha}}{\alpha} \frac{\partial}{\partial x}-\frac{1}{2} \frac{\partial}{\partial u}$, we obtain 


$$
\begin{aligned}
T_{1}^{t}= & c_{1} x^{\alpha-1}\left(-\frac{1}{2}-\frac{x^{1-\alpha} t^{\alpha}}{\alpha} u_{x}\right)-c_{2} \frac{t^{\alpha}}{\alpha} v_{x}, \\
T_{1}^{x}= & \left(-\frac{1}{2}-\frac{x^{1-\alpha} t^{\alpha}}{\alpha} u_{x}\right)\left(c_{1} u-c_{2} v\right) t^{1-\alpha} \\
& -c_{1}\left(\frac{(1-\alpha)}{\alpha} u_{x}+\frac{1}{\alpha} x u_{x x}\right) x^{1-2 \alpha} t^{2 \alpha-1} \\
& +\frac{x^{1-\alpha} t^{\alpha}}{\alpha} v_{x}\left(2 c_{1}+2 c_{2} u+c_{2} x^{-\alpha}\right. \\
& \left.+c_{2}(1-\alpha) x^{-\alpha}\right) t^{\alpha-1} \\
& +c_{2}\left(\frac{(1-\alpha)}{\alpha} v_{x}+\frac{1}{\alpha} \chi v_{x x}\right) x^{1-2 \alpha} t^{2 \alpha-1} .
\end{aligned}
$$

10 obtain

For the symmetry $X_{1}=\frac{t}{\alpha} \frac{\partial}{\partial t}+\frac{x}{2 \alpha} \frac{\partial}{\partial x}-\frac{1}{2} u \frac{\partial}{\partial u}-v \frac{\partial}{\partial v}$, we

$$
\begin{aligned}
T_{1}^{t}= & c_{1} x^{\alpha-1}\left(-\frac{1}{2} u-\frac{x}{2 \alpha} u_{x}-\frac{t}{\alpha} u_{t}\right) \\
& +c_{2} x^{\alpha-1}\left(-v-\frac{x}{2 \alpha} v_{x}-\frac{t}{\alpha} v_{t}\right) \\
T_{1}^{x}= & \left(-\frac{1}{2} u-\frac{x}{2 \alpha} u_{x}-\frac{t}{\alpha} u_{t}\right)\left(c_{1} u+c_{1} \mu c_{2} x^{-\alpha}+c_{2} v\right. \\
& +c_{1} \beta(1-\alpha)(1-2 \alpha) x^{-2 \alpha}-c_{1} \mu(1-\alpha) x^{-\alpha} \\
& \left.-3 c_{2} \beta(1-\alpha)^{2} x^{-\alpha}+c_{2} \beta(2-2 \alpha)(1-\alpha) x^{-\alpha}\right) t^{\alpha-1} \\
& -\left(\frac{1}{2} u_{x}+\frac{x}{2 \alpha} u_{x x}+\frac{1}{2 \alpha} u_{x}+\frac{1}{\alpha} u_{t x}\right) \\
& \times\left(c_{1} \mu x^{1-\alpha}+3 \beta(1-\alpha) c_{2} x^{1-2 \alpha}-c_{2} \beta x^{2-2 \alpha}\right) t^{\alpha-1} \\
& -\left(\frac{1}{2} u_{x x}+\frac{1}{2 \alpha} u_{x x}+\frac{x}{2 \alpha} u_{x x x}+\frac{1}{2 \alpha} u_{x x}+\frac{1}{\alpha} u_{t x x}\right) \\
& \times c_{2} x^{2-2 \alpha} t^{\alpha-1}+\left(-v-\frac{x}{2 \alpha} v_{x}-\frac{t}{\alpha} v_{t}\right) \\
& \times\left(c_{1}+c_{2} u-c_{2} \mu(1-\alpha) x^{1-\alpha}+c_{2} \mu(1-\alpha) x^{-\alpha}\right) t^{\alpha-1} \\
& -\left(v_{x}+\frac{x}{2 \alpha} v_{x x}+\frac{1}{2 \alpha} v_{x}+\frac{1}{\alpha} v_{t x}\right) c_{2} \mu x^{1-\alpha} t^{\alpha-1}
\end{aligned}
$$

$$
\begin{aligned}
T_{1}^{t}= & -c_{1} x^{\alpha-1} t^{1-\alpha} u_{t}-c_{2} x^{\alpha-1} t^{1-\alpha} v_{t}, \\
T_{1}^{x}= & -u_{t}\left(c_{1} u+c_{1} \mu c_{2} x^{-\alpha}+c_{2} v+c_{1} \beta(1-\alpha)\right. \\
& \times(1-2 \alpha) x^{-2 \alpha}-c_{1} \mu(1-\alpha) x^{-\alpha} \\
& -3 c_{2} \beta(1-\alpha)^{2} x^{-\alpha} \\
& \left.+c_{2} \beta(2-2 \alpha)(1-\alpha)\right) x^{-\alpha}-u_{t x}\left(c_{1} \mu x^{1-\alpha}\right. \\
& \left.+3 \beta(1-\alpha) c_{2} x^{1-2 \alpha}-c_{2} \beta x^{2-2 \alpha}\right)-u_{t x x} c_{2} x^{2-2 \alpha} \\
& -v_{t}\left(c_{1}+c_{2} u-c_{2} \mu(1-\alpha) x^{1-\alpha}\right. \\
& \left.+c_{2} \mu(1-\alpha) x^{-\alpha}\right)-c_{2} \mu x^{1-\alpha} v_{t x} .
\end{aligned}
$$

For the symmetry $X_{3}=\frac{x^{1-\alpha} t^{\alpha}}{\alpha} \frac{\partial}{\partial x}+\frac{\partial}{\partial u}$, we obtain

$$
\begin{aligned}
T_{1}^{t}= & c_{1} x^{\alpha-1}\left(-1-\frac{x^{1-\alpha} t^{\alpha}}{\alpha} u_{x}\right)-c_{2} \frac{t^{\alpha}}{\alpha} v_{x} \\
T_{1}^{x}= & \left(-1-\frac{x^{1-\alpha} t^{\alpha}}{\alpha} u_{x}\right)\left(c_{1} u+c_{1} \mu c_{2} x^{-\alpha}+c_{2} v\right. \\
& +c_{1} \beta(1-\alpha)(1-2 \alpha) x^{-2 \alpha}-c_{1} \mu(1-\alpha) x^{-\alpha} \\
& \left.-3 c_{2} \beta(1-\alpha)^{2} x^{-\alpha}+c_{2} \beta(2-2 \alpha)(1-\alpha)\right) x^{-\alpha} t^{\alpha-1} \\
& -\left(\frac{(1-\alpha)}{\alpha} u_{x}+\frac{1}{\alpha} x u_{x x}\right)\left(c_{1} \mu x^{1-\alpha}+3 \beta(1-\alpha) c_{2} x^{1-2 \alpha}\right. \\
& \left.-c_{2} \beta x^{2-2 \alpha}\right) t^{2 \alpha-2}-\left(\frac{(1-\alpha)}{\alpha} u_{x x}+\frac{1}{\alpha} x u_{x x x}+\frac{1}{\alpha} u_{x x}\right) \\
& \times c_{2} x^{2-2 \alpha} t^{\alpha-1}+\frac{x^{1-\alpha} t^{\alpha}}{\alpha} v_{x}\left(2 c_{1}+2 c_{2} u\right. \\
& \left.+c_{2} x^{-\alpha}+c_{2}(1-\alpha) x^{-\alpha}\right) t^{\alpha-1} \\
& +c_{2} \mu\left(\frac{(1-\alpha)}{\alpha} v_{x}+\frac{1}{\alpha} x v_{x x}\right) x^{1-2 \alpha} t^{2 \alpha-1}
\end{aligned}
$$

For the symmetry $X_{4}=x^{1-\alpha} \frac{\partial}{\partial x}$, we obtain 


$$
T_{1}^{t}=-c_{1} u_{x}-c_{2} v_{x},
$$$$
T_{1}^{x}=-\chi^{1-\alpha} u_{x}\left(c_{1} u+c_{1} \mu c_{2} x^{-\alpha}+c_{2} v+c_{1} \beta(1-\alpha)\right.
$$$$
\times(1-2 \alpha) x^{-2 \alpha}-c_{1} \mu(1-\alpha) x^{-\alpha}-3 c_{2} \beta(1-\alpha)^{2}
$$$$
\left.\times x^{-\alpha}+c_{2} \beta(2-2 \alpha)(1-\alpha) x^{-\alpha}\right) t^{\alpha-1}
$$$$
-\left((1-\alpha) x^{-\alpha} u_{x}+x^{1-\alpha} u_{x x}\right)
$$$$
\times\left(c_{1} \mu x^{1-\alpha}+3 \beta(1-\alpha) c_{2} x^{1-2 \alpha}-c_{2} \beta x^{2-2 \alpha}\right) t^{\alpha-1}
$$$$
\text { - }\left(2(1-\alpha) x^{-\alpha} u_{x x}-\alpha(1-\alpha) x^{-\alpha-1} u_{x}\right.
$$$$
\left.+x^{1-\alpha} u_{x x x}\right) c_{2} x^{2-2 \alpha} t^{\alpha-1}-x^{1-\alpha} v_{x}\left(c_{1}+c_{2} u\right.
$$

10

$$
\begin{aligned}
& \left.-c_{2} \mu(1-\alpha) x^{1-\alpha}+c_{2} \mu(1-\alpha) x^{-\alpha}\right) t^{\alpha-1} \\
& -\left((1-\alpha) x^{-\alpha} v_{x}+x^{1-\alpha} v_{x x}\right) c_{2} \mu x^{1-\alpha} t^{\alpha-1} .
\end{aligned}
$$

\section{References}

[1] Al Qurashi MM. Conserved vectors with conformable derivative for certain systems of partial differential equations with physical applications. Open Phys. 2020;18(1):164-9. 